\title{
Ezetimibe prevents myocardial remodeling in an obese rat model by inhibiting inflammation
}

\author{
Xiao-Xing $\mathrm{Li}^{1,3}$, Lang Zhao ${ }^{3}$, Ying Chang ${ }^{2,3}$, Bao-Shan Liu'2,3, Feng $\mathrm{Xu}^{2,3}$, Cheng Zhang ${ }^{3}$, \\ Xiao-Ping $\mathrm{Ji}^{3}$, Yu-Guo Chen²,3 and Chuan-Bao Li ${ }^{2,3 凶}$
}

'Department of Geriatrics, Qilu Hospital, Shandong University Jinan, Shandong, China; 2Department of Emergency, Chest Pain Center, Qilu Hospital, Shandong University, Jinan, Shandong, China; ${ }^{3}$ Key Laboratory of Cardiovascular Remodeling and Function Research, Chinese Ministry of Education and Chinese Ministry of Public Health, Qilu Hospital, Shandong University, Jinan, Shandong, China

\begin{abstract}
Inflammation plays an important role in the development of many obesity-related diseases. This study aimed to investigate the effect of ezetimibe on inflammation and myocardial remodeling in obese rats. A rat model of obesity was established, and myocardial damage was examined by transmission electron microscopy and Masson staining. Twenty obese rats were divided into two groups $(n=10)$ : obese group and ezetimibe group. Ten SD rats were used as controls. Western blot was performed to monitor the expression of P-p38MAPK and interleukin (IL)-6. Immunohistochemical staining was used to monitor the expression of intercellular adhesion molecule-1 and vascular cell adhesion molecule- 1 . In the obese rats group, we observed increased inflammatory factors and myocardial hypertrophy. In contrast, the ezetimibe group exhibited decreased expression of inflammatory factors and an improvement in myocardial remodeling compared to the obese group. Mechanistically, we found that ezetimibe decreased P-p38MAPK, IL6 , intercellular adhesion molecule-1, and vascular cell adhesion molecule- 1 levels in the hearts of the obese rats. Taken together, these results indicate that ezetimibe may improve myocardial remodeling in obese rats by inhibiting inflammation.
\end{abstract}

Key words: obese, inflammation, remodeling, ezetimibe, IL-6

Received: 01 April, 2018; revised: 28 May, 2018; accepted: 28 May, 2018; available on-line: 08 September, 2018

更-mail: bao2460@126.com

Abbreviations: CVD, cardiovascular disease; CRP, C-reactive protein; ICAM-1, intercellular adhesion molecule-1; EF, ejection fraction; FS, fractional shortening; $\mathrm{HF}$, high fat; $\mathrm{HDL}-\mathrm{C}$, high-density lipoprotein-cholesterol; IR, insulin resistance; IRS-1, insulin receptor substrate 1; IL-6, Interleukin 6; LDL-C, low-density lipoprotein-cholesterol; LVEDD, left ventricular end-diastolic diameter; LVESD, left ventricular end-systolic diameter; TEM, transmission electron microscope; TG, triglycerides; MCP-1, macrophage chemoattractant protein 1; VCAM-1, vascular cell adhesion molecule-1

\section{INTRODUCTION}

The combination of abdominal obesity and impaired glucose and lipid metabolism place individuals at a significantly increased risk of developing cardiovascular disease (CVD) (Kim et al., 2017; Larsson et al., 2017). Moreover, changes in the macro-vascular structure and myocardial function in obese patients may contribute to the development of CVD. In obese patients, there is increased myocardial remodeling and dysfunction, which are predictive of early pathological changes in the heart (Alpert et al., 2016; Gu et al., 2015). Given the high morbidity and mortality of CVD in obese patients (Liao et al., 2014), it is critically important to understand the mechanisms underlying myocardial remodeling to delay the progression of CVD using multifactorial intervention strategies and decrease cardiovascular end-point events in patients with obesity.

Inflammatory reactions have been shown to contribute to myocardial remodeling, and interleukin 6 (IL-6) is a factor that can function as both a pro-inflammatory cytokine and an anti-inflammatory myokine (González et al., 2015; Pedersen et al., 2006). Previous studies have shown that patients with obesity display increased levels of C-reactive protein (CRP) and IL- 6 compared to the controls (Ellulu et al., 2016; Okamoto et al., 2015). In addition, the activation of p38MAPK has been correlated with myocardial remodeling (Yoshida et al., 2001; Baraka et al., 2009; Yu et al., 2012). p38MAPK can be activated by a variety of stimuli, such as elevated levels of free fatty acids, cholesterol, glucose, and proinflammatory mediators (Ragheb et al., 2009; Shalini et al., 2016). Moreover, the activation of p38MAPK can regulate gene expression, which may promote myocardial remodeling (Baraka et al., 2009).

Ezetimibe, a drug that inhibits the absorption of cholesterol from the small intestine and decreases circulating cholesterol levels, attracted recent attention because of its noted anti-inflammatory effects (Kuhlencordt et al., 2009; Gómez-Garre et al., 2009). However, whether the anti-inflammatory properties of ezetimibe can attenuate myocardial remodeling remains unknown. This study aimed to investigate the effects of ezetimibe on improving inflammation and myocardial remodeling. Using obese rats induced by a high fat (HF) diet as an animal model of obesity, we demonstrated that ezetimibe can effectively reduce the expression of inflammatory factors and improve myocardial remodeling in obese rats.

\section{MATERIALS AND METHODS}

Animal model. All experimental protocols involving animals were performed in line with the National Institutes of Health and care and use of laboratory animals of Shandong University. An animal housing room was used to house the rats under controlled temperature $\left(23-25^{\circ} \mathrm{C}\right)$, humidity, and $14 \mathrm{~h}$ light $/ 10 \mathrm{~h}$ dark cycle throughout the entire experimental period.

A total of 40 male SD rats (8-week-old) were randomly divided into a control group $(\mathrm{n}=10)$ and model group $(n=30)$. The control rats were fed a standard chow and tap water. The rats in the model group were fed a highfat diet (HF-diet). After 16 weeks, 20 SD rats had developed obesity. The 20 obese rats were further divid- 
Table 1. Metabolic variables at the end of the experiment

\begin{tabular}{|c|c|c|c|}
\hline & $N C(n=10)$ & $O B(n=10)$ & OB+Ezetimibe $(n=10)$ \\
\hline Body weight (g) & $494.96 \pm 21.95$ & $654.64 \pm 67.54^{* *}$ & $514.90 \pm 46.75^{+\dagger}$ \\
\hline Cholesterol (mmol/L) & $1.98 \pm 0.11$ & $2.93 \pm 0.04^{* *}$ & $2.07 \pm 0.11^{\dagger+}$ \\
\hline Triglycerides (mmol/L) & $0.95 \pm 0.08$ & $1.56 \pm 0.08^{* *}$ & $0.98 \pm 0.06^{t \dagger}$ \\
\hline $\mathrm{HDL}-\mathrm{C}(\mathrm{mmol} / \mathrm{L})$ & $1.05 \pm 0.07$ & $0.72 \pm 0.05^{*}$ & $1.09 \pm 0.06^{t \dagger}$ \\
\hline LDL-C (mmol/L) & $0.74 \pm 0.04$ & $1.52 \pm 0.06^{* *}$ & $0.94 \pm 0.05^{t \dagger}$ \\
\hline
\end{tabular}

Values are expressed as the mean \pm SEM. ${ }^{*} P<0.05 ;{ }^{* *} P<0.01$ compared to the NC group. ${ }^{++} P<0.01$ compared to the OB group.

ed into two groups: 1) the obesity (OB) group ( $n=10)$, which received a continued $\mathrm{HF}$-diet; 2) the ezetimibe treated $(\mathrm{OB}+$ Ezetimibe) group $(\mathrm{n}=10)$, which received a continued HF-diet plus treatment with ezetimibe at 10 $\mathrm{mg} / \mathrm{kg} /$ day by gavage. Rats in both the control and OB groups were given the same volume of saline by gavage. The treatment period lasted for eight weeks.

Body weight (BW) was measured in the morning on a weekly basis throughout the experimental period. Venous blood samples were collected after $12 \mathrm{~h}$ fasting and the serum concentration of triglycerides (TG), cholesterol, high-density lipoprotein-cholesterol (HDL-C), low-density lipoprotein-cholesterol (LDL-C), and inflammatory factors were quantified by the Department Clinical Laboratory (Qilu Hospital affiliated with Shandong University, Jinan, China). At the end of the experimental period, rats were sacrificed by an over-dose of pentobarbital and the hearts were aseptically excised for subsequent analysis.

Echocardiographic examination. An echocardiographic examination was performed using a Vevo 770 cardiac system (Visual Sonics Inc., Toronto, Canada) under anesthesia with $10 \%$ chloral hydrate $(0.3 \mathrm{~mL} / 100 \mathrm{~g})$. Left ventricular (LV) end-diastolic and end-systolic diameters, posterior wall and septum thickness, fractional shortening (FS), and the ejection fraction (EF) were measured according to the American Society of Echocardiography guidelines (Sahn et al., 1978). Doppler measurements included peak early (E) and late (A) mitral valve inflow velocities and E/A ratio. For all echocardiographic measurements, the values of three consecutive cardiac cycles were averaged.

Ultrastructural observations. The left ventricular tissues (approximately $0.5 \times 1 \times 0.5 \mathrm{~mm}$ ) from each group were fixed overnight in $2 \%$ glutaraldehyde and washed with $0.2 \mathrm{~mol} / \mathrm{L}$ phosphate buffer three times. The samples were then fixed with $1 \%$ osmium tetraoxide, washed again with $0.2 \mathrm{~mol} / \mathrm{L}$ phosphate buffer, and dehydrated in different concentrations of ethanol. The ultrastructure of the cardiac muscle was observed using a transmission electron microscope (TEM; H-7000FA, Hitachi, Tokyo, Japan).

Histological analysis. Briefly, the lower part of the left ventricular tissue was fixed in $4 \%$ formaldehyde and embedded in paraffin, before being cut into $5 \mu \mathrm{m}$ thick cross-sections. The sections were dewaxed and rehydrated, and antigen retrieval by microwaving was performed. The sections were stained with hematoxylin-eosin staining and Masson Accustain Trichrome stain (Sigma, St. Louis, MO, USA).

Western blot analysis. The heart tissues were lysed and $50 \mu \mathrm{g}$ of protein was resolved on $10 \%$ SDSpolyacrylamide gels and transferred onto nitrocellulose membranes. Membranes were blocked at $4^{\circ} \mathrm{C}$ in $5 \%$ nonfat milk dissolved in Tris-buffered saline
(25 mM Tris, $137 \mathrm{mM} \mathrm{NaCl}$, and $2.7 \mathrm{mM} \mathrm{KCl}$ ) containing $0.05 \%$ Tween-20 and then incubated with Pp38MAPK (rabbit, 1:400) or IL-6 (rabbit, 1:500) primary antibodies at $4^{\circ} \mathrm{C}$ overnight. The membranes were washed three times in TBS-T and incubated with a horseradish peroxidase-conjugated goat anti-rabbit secondary antibody at room temperature for $2 \mathrm{~h}$. Immunoreactive bands were visualized using an enhanced chemiluminescence kit and quantified with an image analyzer (AlphaImager 2200, USA). Protein levels were normalized to $\beta$-actin.

Immunohistochemical staining. Paraffin-embedded $5 \mu \mathrm{m}$ thick ventricular tissue sections were rehydrated, and antigen retrieval was performed by an incubation in $1 \%$ citrate buffer $\left(\mathrm{pH}\right.$ 6.0) at $92-98^{\circ} \mathrm{C}$ for $15 \mathrm{~min}$. The sections were then incubated in PBS containing $3 \%$ hydrogen peroxide to quench any endogenous peroxidase activity. Next, the sections were blocked in goat serum for $30 \mathrm{~min}$, followed by an incubation with VCAM-1 and ICAM-1 primary antibodies (Abcam, USA) overnight at $4^{\circ} \mathrm{C}$. The negative controls were incubated with PBS instead of the primary antibody. A secondary conjugated IgG antibody was then added and the sections were incubated for $1 \mathrm{~h}$ at $37^{\circ} \mathrm{C}$. DAB substrate kits were applied and the sections were viewed under a confocal FV 1000 SPD Laser Scanning microscope (Olympus, Japan). Three sections per rat and four areas from each ventricular section were analyzed.

Statistical analysis. All values are presented as the mean \pm S.E.M. The results were compared using a oneway ANOVA, followed by a Tukey-Kramer post-hoc test. All statistical analyses were performed using SPSS 17.0 software, and a threshold value of $P<0.05$ was considered significant.

Table 2. Parameters measured by echocardiogram at the end of the study

\begin{tabular}{llcc}
\hline & NC $(n=10)$ & OB $(n=10)$ & OB+Ezetimibe $(n=10)$ \\
\hline LVEDD & $4.06 \pm 0.57$ & $4.43 \pm 0.30$ & $4.53 \pm 0.75$ \\
\hline LVESD & $1.82 \pm 0.18$ & $1.93 \pm 0.22$ & $2.05 \pm 0.29$ \\
\hline IVS & $2.22 \pm 0.47$ & $3.40 \pm 0.22^{* *}$ & $2.89 \pm 0.26^{+\dagger}$ \\
\hline LVPW & $3.14 \pm 0.44$ & $4.53 \pm 0.80^{* *}$ & $3.45 \pm 0.52^{+\dagger}$ \\
\hline FS & $0.61 \pm 0.03$ & $0.59 \pm 0.04$ & $0.60 \pm 0.05$ \\
\hline E/A & $2.13 \pm 0.19$ & $1.45 \pm 0.21^{* *}$ & $1.61 \pm 0.22^{+}$ \\
\hline
\end{tabular}

All data are mean \pm S.E.M. LVEDD $(\mathrm{mm})$ : left ventricular end-diastolic dimension; LVESD ( $\mathrm{mm}$ ): left ventricular end-systolic dimension; IVS $(\mathrm{mm})$ : intraventricular septal wall; LVPW $(\mathrm{mm})$ : left ventricular posterior wall thickness; FS: fractional shortening; E/A: ratio of peak early diastolic filling velocity to peak velocity at atrial contractions. ${ }^{*} P<0.01$; ${ }^{*} P<0.05$ compared to the NC group; ${ }^{++} P<0.01,+P<0.05$ compared to the OB group. 


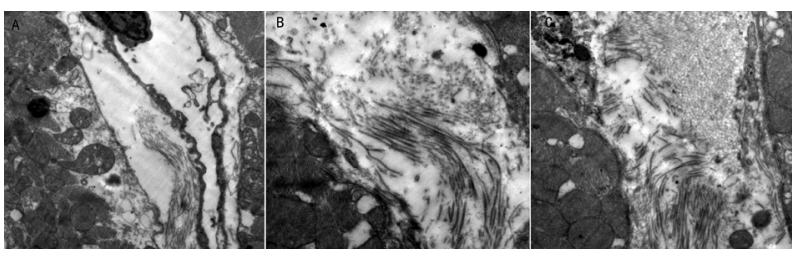

Figure 1. Representative photomicrographs of the transmission electron microscopy analysis of the myocardium of rats.

(A) NC group $(n=10)$ : deposition of collagen fibers in the extracellular matrix was clear and normal, and the mitochondria appeared to be of normal size and number; (B) OB group $(n=10)$ : cardiac myocytes exhibited an excessive deposition of collagen fibers in the extracellular matrix, and the mitochondria appeared to be swollen; (C) OB+Ezetimibe group $(n=10)$ : the mitochondria appeared to be of normal size, and deposition of collagen fibers in the extracellular matrix was decreased compared to the OB group. $($ TEM $\times 10000)$ concentric hypertrophy $(P<0.05)$. FS, representing $L V$ systolic function, did not differ between the HF-fed and $\mathrm{NC}$ rats $(P>0.05)$. However, E/A was decreased significantly in the $\mathrm{OB}$ rats compared to the $\mathrm{NC}$ rats $(P<0.05)$ (Table 2). At the end of the study period, there were more marked changes in IVS, LVPW, and $\mathrm{E} / \mathrm{A}$ in the $\mathrm{OB}$ rats (Table 2). Additionally, when compared to the $\mathrm{OB}$ rats, treatment with ezetimibe slowed the progression of cardiac hypertrophy and protected the diastolic function (Table 2).

\section{Ezetimibe improved the ultrastructure of cardiomyocytes in obese rats}

TEM analysis showed increased collagen fiber and mitochondria swelling in the cardiomyocytes of $\mathrm{OB}$ rats compared to the NC rats (Fig. 1). Moreover, after
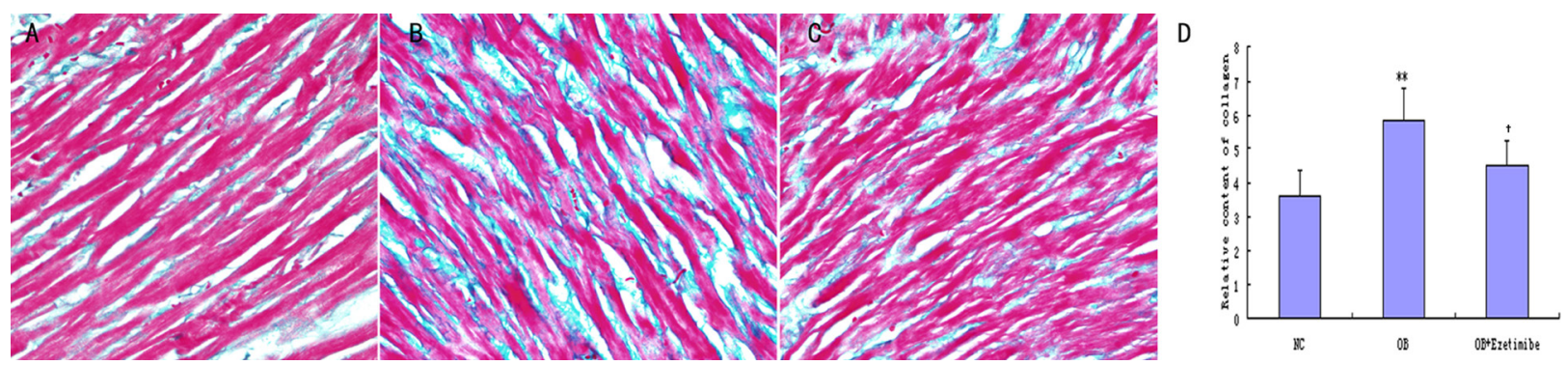

Figure 2. Masson's Trichrome staining of myocardial tissues.

(A) Normal SD rats showed normal quantity and the distribution of collagen fibers (400X). (B) OB rats displayed hyperplastic and disorganized collagen fibers (400x). (C) OB rats treated with Ezetimibe exhibited almost normal appearance of collagen fibers (400x). (D) Comparison of the relative content of collagen in three groups of rats. ${ }^{* *} P<0.01$ vs. NC group; ${ }^{+} P<0.05$ vs. OB group. NC group ( $n=10$ ); OB group $(n=10) ; O B+$ Ezetimibe group $(n=10)$.

\section{RESULTS}

\section{Ezetimibe affected baseline metabolism in obese rats}

An obese rat model was established by feeding male SD rats with a HF diet for 16 weeks. After 16 weeks, the obese rats were found to develop hypercholesterolemia, lower HDL-C, and obesity compared to the normal control $(\mathrm{NC})$ group $(P<0.01)$. After eight weeks of treatment with ezetimibe, cholesterol, TG, and BW were significantly decreased in $\mathrm{OB}+$ Ezetimibe group compared to the $\mathrm{OB}$ rats (Table 1$)$.

\section{Ezetimibe improved cardiac function in obese rats}

After 16 weeks, interventricular septal (IVS) and LV posterior wall (LVPW) thickness in the OB rats were substantially increased, indicating the presence of LV

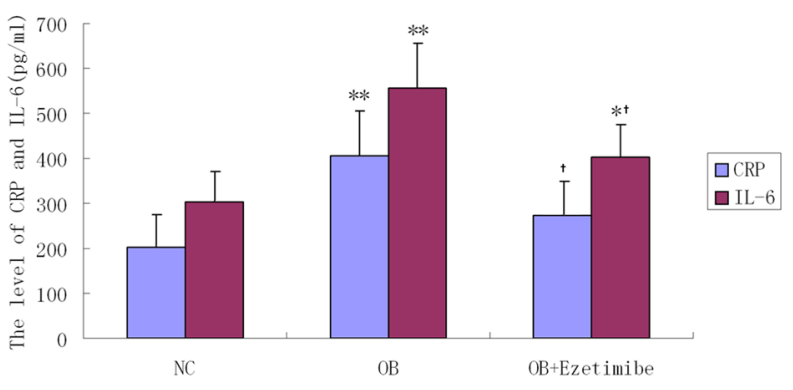

Figure 3. Comparison of serum CRP and IL-6 levels.

Data were shown as the means \pm S.E.M., ${ }^{*} P<0.01$ vs. NC group, ${ }^{+} P<0.01$ vs. OB group. NC group $(\mathrm{n}=10) ; \mathrm{OB}$ group $(\mathrm{n}=10) ;$ $\mathrm{OB}+$ Ezetimibe group $(\mathrm{n}=10)$. ezetimibe treatment, the cardiac ultrastructure was obviously improved (Fig. 1).

\section{Ezetimibe reduced the collagen content in obese rats}

Masson staining revealed an increase of interstitial fibrosis in the myocardium of $\mathrm{OB}$ rats compared to the NC group. However, interstitial fibrosis was reduced after ezetimibe treatment (Fig. 2).

\section{Ezetimibe decreased serum CRP and IL- 6 levels in obese rats}

Serum CRP and IL-6 levels of the OB rats were significantly higher than that of the NC rats. However, serum levels of CRP and IL-6 decreased significantly in the ezetimibe-treated group compared to the OB group (Fig. 3).

\section{Ezetimibe decreased P-p38MAPK and IL-6 levels in the hearts of obese rats}

The levels of P-p38MAPK and IL-6 in the rat hearts were evaluated by Western blot. In the $\mathrm{OB}$ rats, the levels of P-p38MAPK and IL-6 were significantly increased in the myocardial tissues compared to the NC group. However, both P-p38MAPK and IL-6 levels decreased significantly in ezetimibe-treated rats when compared to the $\mathrm{OB}$ rats (Fig. 4).

\section{Ezetimibe decreased ICAM-1 and VCAM-1 levels in the hearts of obese rats}

In the OB rats, ICAM-1 and VCAM-1 levels in the hearts were significantly higher compared to the $\mathrm{NC}$ group. However, the levels of ICAM-1 and VCAM-1 


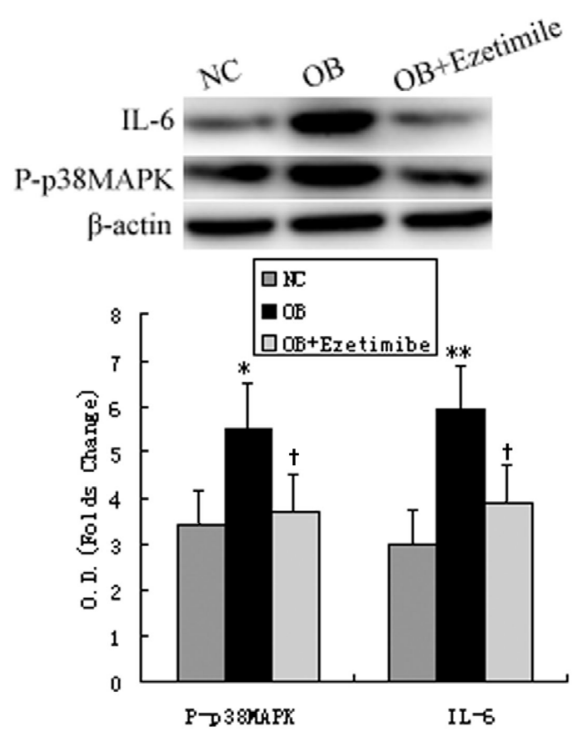

Figure 4. Western blot analysis of P-p38MAPK and IL-6 levels in the myocardial tissues of rats.

Representative blots were shown. The bar graph displays the relative protein levels. Each bar represents the mean \pm SEM. ${ }^{* *} P<0.01$; ${ }^{*} P<0.05$ vs. NC group; ${ }^{+} P<0.05$ vs. OB group. NC group $(n=10) ; O B$ group $(n=10) ; O B+$ Ezetimibe group $(n=10)$.

were significantly lower in the $\mathrm{OB}+$ ezetimibe group compared to the OB group (Fig. 5).

\section{DISCUSSION}

In this study, a rat model of obesity was used to demonstrate that obesity-enhanced cardiac inflammation and remodeling could be inhibited by treatment with ezetimibe. Mechanistically, we found that obesity led to the activation of p38MAPK and enhanced the expression of inflammatory factors, such as IL-6, ICAM-1, and VCAM-1. Treatment with ezetimibe inhibited such obesity-induced activation of p38MAPK and the upregulation of several inflammatory factors.

Obesity is one known risk factor for CVD, for which the underlying mechanism is thought to be related to lipotoxicity and insulin resistance (IR) (Avalos-Soriano et al., 2016). In the present study, HF-fed rats developed obesity with low levels of HDL-C, as well as elevated cholesterol, TG, and BW. Moreover, most obese patients already exhibit vascular abnormalities by the time they are diagnosed with a metabolic disorder (Ruderman \& Schneider, 1992; Ridker et al., 2003). In our study, the obese rat model showed enhanced myocardial remodeling and inflammation, all of which may contribute to the high prevalence of cardiovascular complications associated with obesity. Moreover, the risk factors associated with CVD, including dyslipidemia, hypertension, and hyperglycemia, are considered to be initiation and progression factors of myocardial remodeling. In addition, inflammation is a hallmark sign throughout the distinct stages of myocardial remodeling induced by a HF-fed diet (Klingenberg \& Luscher, 2012; Haffner, 2006). Furthermore, a systemic chronic inflammatory response in obesity, characterized by altered cytokine production and the activation of inflammatory signaling pathways, is another important mechanism associated with the initiation and progression of myocardial remodeling (MartínezMartínez et al., 2016; Mayerl et al., 2006).

IL-6 and CRP are the major mediators of inflammation (Young et al., 2014; Libinaki et al., 2010; Hattori et al., 2003). In addition, the p38MAPK pathway is a key
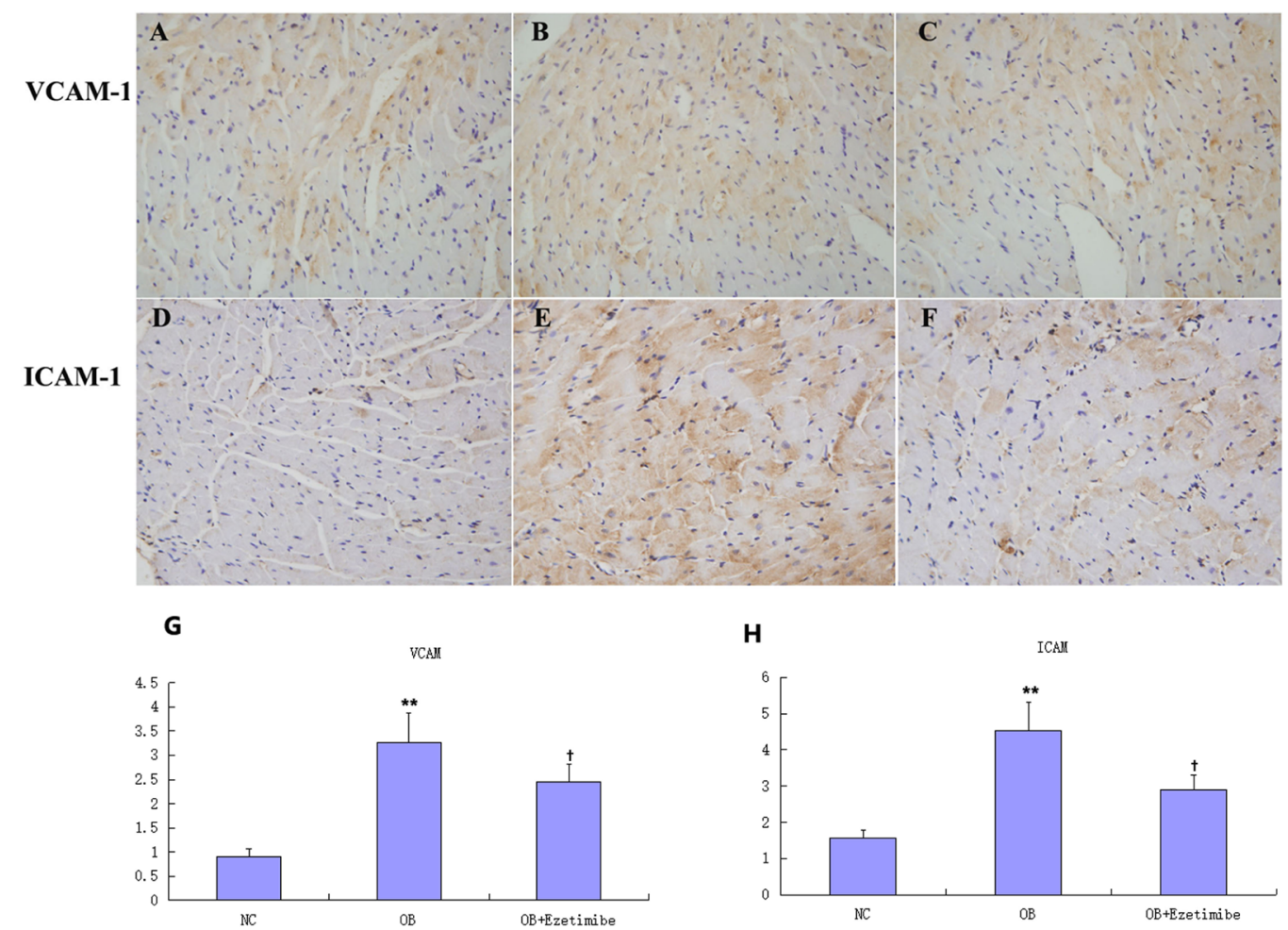

Figure 5. Immunohistochemistry staining of VCAM-1 and ICAM-1 in the myocardial tissues of the rats.

$(\mathbf{A}$ and E) NC group $(n=10)$; (B and F) OB group $(n=10) ;(C$ and $\mathbf{G})$ OB+Ezetimibe group $(n=10)$. Representative micrographs of immunohistochemistry staining were shown. Original magnification: $\times 400$. (D and H) Quantitative analysis of VCAM-1and ICAM-1 staining. Data are presented as the mean \pm S.E.M. ${ }^{* *} P<0.01$ vs. the NC group; ${ }^{++} P<0.01$ vs. the OB group. 
regulator of pro-inflammatory cytokine biosynthesis, and its components are potential targets for the treatment of inflammatory diseases associated with obesity (Cuenda \& Rousseau, 2007). It is interesting to note that p38MAPK activation is essential for VCAM-1 and ICAM-1 expression in cardiac cells (Kacimi et al., 1998). VCAM-1 and ICAM-1 are important factors that promote inflammation. In this study, the cardiac tissue of obese rats exhibited: cardiomyocytes with an abnormal ultrastructure; increased interstitial fibrosis; thicker walls; reduced diastolic function; p38MAPK activation; and increased expression of CRP, IL-6, VCAM-1, and ICAM-1. Previous studies have indicated that dyslipidemia induces the formation of atherosclerotic lesions by increasing the release of inflammatory molecules, such as TNF- $\alpha$ and IL-6 (Derosa et al., 2011; Li et al., 2015). These results suggest that the obesity-induced activation of p38MAPK may promote cardiac inflammation and plays an important role in the pathogenesis of cardiac remodeling.

Recent studies suggested that ezetimibe may inhibit inflammation and improve metabolic functionality (Shapiro \& Fazio, 2016; et al., 2015). Ezetimibe displays cardioprotective activity by inhibiting the expression of vascular CD14, a marker of the infiltration of mononuclear cells (Kuhlencordt et al., 2009). Ezetimibe also interferes with the activity of nuclear factor- $x \mathrm{~B}$ in leukocytes and reduces the amount of monocyte and macrophage chemoattractant protein 1 (MCP-1) (Gómez-Garre et al., 2009). In addition, the anti-inflammatory effects of ezetimibe are thought to have a favorable impact on lipid metabolism (Krysiak et al., 2014); however, no studies have assessed the effect of ezetimibe on myocardial remodeling after a high fat diet. Therefore, we investigated the effects of ezetimibe on inflammation and myocardial remodeling in rats with obesity induced by a HF diet. We found that ezetimibe could effectively alleviate myocardial remodeling by inhibiting the level of inflammation. In addition to lowering lipid metabolism, ezetimibe prevented cardiac inflammation and collagen deposition induced by a HF diet.

Taken together, our findings suggest that the upregulation of inflammatory factors promotes myocardial remodeling in an obese rat model, which may be due to the activation of p38MAPK in the heart tissue. Treatment with ezetimibe may have the potential to inhibit p38MAPK activation, reduce the expression of inflammatory factors, and alleviate myocardial remodeling associated with obesity.

\section{Conflict of interests}

The authors confirm that there are no conflicts of interest.

\section{Acknowledgements of Financial Support}

This work was supported by the National Natural Science Foundation of China (81400282 and 81570401), the Taishan Scholar Program of Shandong Province (ts20130911 and tsqn20161065), and the Postdoctoral Science Foundation (2017M612293 and 201402033).

\section{REFERENCE}

Alpert MA, Omran J, Bostick BP (2016) Effects of obesity on cardiovascular hemodynamics, cardiac morphology, and ventricular function. Curr Obes Rep 5: 424-434. doi: 10.1007/s13679-016-0235-6

Avalos-Soriano A, De la Cruz-Cordero R, Rosado JL, Garcia-Gasca $\mathrm{T}$ (2016) 4-Hydroxyisoleucine from fenugreek (Trigonella foenumgraecum): effects on insulin resistance associated with obesity. Molecules 21: 11. doi: 10.3390/molecules21111596
Baraka A, Mikhail M, Guemei A, El Ghotny S (2009) Effect of targeting mitogen-activated protein kinase on cardiac remodeling in rats. J Cardiovasc Pharmacol Ther 14: 339-346. doi: $10.1177 / 1074248409349620$

Cuenda A, Rousseau S (2007) p38 MAP-kinases pathway regulation, function and role in human diseases. Biochim Biophys Acta 1773: 1358-1375. doi: 10.1016/j.bbamcr.2007.03.010

Derosa G, Maffioli P, Ferrari I, Fogari E, D'Angelo A, Palumbo I, Randazzo S, Bianchi L, Cicero AF (2011) Acarbose actions on insulin resistance and inflammatory parameters during an oral fat load. Eur I Pharmacol 651: 240-250. doi: 10.1016/j.ejphar.2010.11.015

Ellulu MS, Khaza'ai H, Rahmat A, Patimah I, Abed Y (2016) Obesity can predict and promote systemic inflammation in healthy adults. Int J Cardiol 215: 318-324. doi: 10.1016/j.ijcard.2016.04.089

Gómez-Garre D, Muñoz-Pacheco P, González-Rubio ML, Aragoncillo P, Granados R, Fernández-Cruz A (2009)Ezetimibe reduces plaque inflammation in a rabbitmodel of atherosclerosis and inhibits monocyte migration in addition to its lipid-lowering effect. Brit J Pharmacol 156: 1218-1227. doi: 10.1111/j.1476-5381.2008.00091.x

González GE, Rhaleb NE, D’Ambrosio MA, Nakagawa P, Liu Y, Leung P, Dai X, Yang XP, Peterson EL, Carretero OA (2015) Deletion of interleukin- 6 prevents cardiac inflammation, fibrosis and dysfunction without affecting blood pressure in angiotensin II-high salt-induced hypertension. J Hypertens 33: 144-152. doi: 10.1097/ HJH.0000000000000358

Gu H, Liu Y, Mei S, Wang B, Sun G, Wang X, Xiao Y, Staup M, Gregoire FM, Chng K, et al (2015) Left ventricular diastolic dysfunction in nonhuman primate model of dysmetabolism and diabetes. BMC Cardiovasc Disord 15: 141. doi: 10.1186/s12872-015-0133-y

Haffner SM (2006) The metabolic syndrome: inflammation, diabetes mellitus, and cardiovascular disease. Am J Cardiol 97: 3A-11A. doi: 10.1016/j.amjcard.2005.11.010

Hattori Y, Matsumura M, Kasai K (2003) Vascular smooth muscle cell activation by C-reactive protein. Cardiovasc Res 58: 186-195. doi: 10.1016/S0008-6363(02)00855-6

Kacimi R, Karliner JS, Koudssi F, Long CS (1998) Expression and regulation of adhesion molecules in cardiac cells by cytokines: response to acute hypoxia. Circ Res 82: 576-586. doi: 10.1161/01. RES.82.5.576

Kim TJ, Shin HY, Chang Y, Kang M, Jee J, Choi YH, Ahn HS, Ahn SH, Son HJ, Ryu S (2017) Metabolically healthy obesity and the risk for subclinical atherosclerosis. Atherosclerosis 262: 191-197. doi: 10.1016/j.atherosclerosis.2017.03.035

Klingenberg R, Luscher TF (2012) Inflammation in coronary artery disease and acute myocardial infarction - is the stage set for novel therapies? Curr Pharm Des 18: 4358-4369. doi: 10.2174/138161212802481219

Krysiak R, Żmuda W, Okopień B (2014) The effect of ezetimibe on adipose tissue hormones in patients with isolated hypercholesterolemia. Pharmacol Rep 66: 442-447. doi: 10.1016/j. pharep.2014.03.006

Kuhlencordt PJ, Padmapriya P, Rützel S, Schödel J, Hu K, Schäfer A, Huang PL, Ertl G, Bauersachs J (2009) Ezetimibe potently reduces vascular inflammation and arteriosclerosis in eNOS-deficient ApoE ko mice. Atherosclerosis 202: 48-57. doi: 10.1016/j.atherosclerosis.2008.03.021

Larsson SC, Wolk A, Håkansson N, Bäck M (2017) Overall and abdominal obesity and incident aortic valve stenosis: two prospective cohort studies. Eur Heart J 38: 2192-2197. doi: 10.1093/eurheartj/ ehx140

Liao D, Rodríguez-Colón SM, He F, Bixler EO (2014) Childhood obesity and autonomic dysfunction: risk for cardiac morbidity and mortality. Curr Treat Options Cardiovasc Med 16: 342. doi: 10.1007/s11936014-0342-1

Libinaki R, Tesanovic S, Heal A, Nikolovski B, Vinh A, Widdop RE, Gaspari TA, Devaraj S, Ogru E (2010) Effect of tocopheryl phosphate on key biomarkers of inflammation: Implication in the reduction of atherosclerosis progression in a hypercholesterolaemic rabbit model. Clin Exp Pharmacol Physiol 37: 587-592. doi: 10.1111/j.1440-1681.2010.05356.x

Li XX, Li CB, Xiao J, Gao HQ, Wang HW, Zhang XY, Zhang C, Ji XP (2015) Berberine attenuates vascular remodeling and inflammation ina rat model of metabolic syndrome. Biol Pharm Bull 38: 862-868. doi: 10.1248/bpb.b14-00828

Martínez-Martínez E, López-Ándres N, Jurado-López R, Rousseau E, Bartolomé MV, Fernández-Celis A, Rossignol P, Islas F, Antequera A, Prieto S, et al (2015) Galectin-3 participates in cardiovascular remodeling associated with obesity. Hypertension 66: 961-969. doi: 10.1161/HYPERTENSIONAHA.115.06032

Mayerl C, Lukasser M, Sedivy R, Niederegger H, Seiler R, Wick G (2006) Atherosclerosis research from past to present on the track of two pathologists with opposing views, Carl von Rokitansky and Rudolf Virchow. Virchows Arch 449: 96-103. doi: 10.1007/s00428006-0176-7

Okamoto LE, Raj SR, Gamboa A, Shibao CA, Arnold AC, Garland EM, Black BK, Farley G, Diedrich A, Biaggioni I (2015) Sympa- 
thetic activation is associated with increased IL-6, but not CRP in the absence of obesity: lessons from postural tachycardia syndrome and obesity. Am J Physiol Heart Circ Physiol 309: H2098-H2107. doi: 10.1152/ajpheart.00409.2015

Pedersen BK (2006) The anti-inflammatory effect of exercise: its role in diabetes and cardiovascular disease control. Essays Biochem 42: 105-117. doi: 10.1042/bse0420105

Ragheb R, Shanab GM, Medhat AM, Seoudi DM, Adeli K, Fantus IG (2009) Free fatty acid-induced muscle insulin resistance and glucose uptake dysfunction: evidence for PKC activation and oxidative stress-activated signaling pathways. Biochem Biophys Res Commun 389: 211-216. doi: 10.1016/j.bbrc.2009.08.106

Ridker PM, Buring JE, Cook NR, Rifai N (2003) C-reactive protein, the metabolic syndrome, and risk of incident cardiovascular events: an 8-year follow-up of 14719 initially healthy American women. Circulation 107: 391-397. doi: 10.1161/01.CIR.0000055014.62083.05

Ruderman NB, Schneider SH (1992) Diabetes, exercise, and atherosclerosis. Diabetes Care 15: 1787-1793. doi: 10.2337/diacare.15.11.1787

Sahn DJ, DeMaria A, Kisslo J, Weyman A (1978) Recommendations regarding quantitation in M-mode echocardiography: results of a survey of echocardiographic measurements. Circulation 58: 10721083. doi: 10.1161/01.CIR.58.6.1072

Shalini V, Pushpan CK, G S, A J, A H (2016) Tricin, flavonoid from Njavara reduces inflammatory responses in hPBMCs by modulating the $\mathrm{p} 38 \mathrm{MAPK}$ and PI3K/Akt pathways and prevents inflammation associated endothelial dysfunction in HUVECs. Immunobiology 221: 137-144. doi: 10.1016/j.imbio.2015.09.016

Shapiro MD, Fazio S (2016) From lipids to inflammation: new approaches to reducing atherosclerotic risk. Circ Res 118: 732-749. doi: 10.1161/CIRCRESAHA.115.306471

Tie C, Gao K, Zhang N, Zhang S, Shen J, Xie X, Wang JA (2015) Ezetimibe attenuates atherosclerosis associated with lipid reduction and inflammation inhibition. PLoS One 10: e0142430. doi: 10.1371/ journal.pone.0142430

Yoshida K, Yoshivama M, Omura T, Nakamura Y, Kim S, Takeuchi K, Iwao H, Yoshikawa J (2001) Activation of mitogen-activated protein kinases in the non-ischemic myocardium of an acute myocardial infarction in rats. Jpn Circ J 65: 808-814. doi:10.1253/ jcj. 65.808

Young JJ, Bruno D, Pomara N (2014) A review of the relationship between proinflammatory cytokines and major depressive disorder. $J$ Affect Disord 169: 15-20. doi: 10.1016/j.jad.2014.07.032

Yu M, Zheng Y, Sun HX, Yu DJ (2012) Inhibitory effects of enalaprilat on rat cardiac fibroblast proliferation via ROS/P38MAPK/ TGF-31 signaling pathway. Molecules 17: 2738-2751. doi: 10.3390/ molecules17032738 